Research Article

\title{
Effect of Silver Nanoparticles on the Morphology of Toxoplasma gondii and Salmonella braenderup
}

\author{
Diego Vergara-Duque, ${ }^{1}$ Liliana Cifuentes-Yepes, ${ }^{1}$ Tatiana Hincapie-Riaño, ${ }^{1}$ \\ Felipe Clavijo-Acosta, ${ }^{1}$ Graciela Juez-Castillo $\mathbb{D}^{1,},{ }^{1,2}$ and Brayan Valencia-Vidal $\mathbb{I D}^{1,2}$
}

${ }^{1}$ GIPS, Bioengineering, Universidad El Bosque, 110121 Bogotá, Colombia

${ }^{2}$ Oxiris \& Bioaxis Research Group, Bioengineering, Universidad El Bosque, 110121 Bogotá, Colombia

Correspondence should be addressed to Brayan Valencia-Vidal; bavalenciav@unbosque.edu.co

Received 23 April 2020; Accepted 10 June 2020; Published 13 July 2020

Academic Editor: Paresh Chandra Ray

Copyright ( 2020 Diego Vergara-Duque et al. This is an open access article distributed under the Creative Commons Attribution License, which permits unrestricted use, distribution, and reproduction in any medium, provided the original work is properly cited.

\begin{abstract}
The study of silver nanoparticles (AgNPs) has recently increased due to the different antimicrobial properties that have been evaluated. Studies have shown that AgNPs decrease the cell viability of some parasitic species and inhibit bacterial growth and biofilm formation. Toxoplasma gondii is a parasite with different stages of development including the oocyst, and it can survive in the environment for a long time generating contamination of vegetables and water. This parasite has the ability to generate congenital toxoplasmosis and chorioretinitis in humans. Another human pathogen present in water is Salmonella braenderup, this bacterium, when consumed, causes gastroenteritis and typhoid fever. We evaluate the affectation that causes the AgNPs in oocysts of T. gondii and S. braenderup using fluorescence microscopy and scanning electron microscopy techniques. The results showed that at different ratios of AgNPs and microorganisms, as well as at different exposure time during the treatments, morphological alteration of the cell structure of oocysts of $T$. gondii and $S$. braenderup was evidenced, suggesting a potential treatment method for the inhibition of the viability of these microorganisms.
\end{abstract}

\section{Introduction}

Water is a fundamental element for life and must have suitable potability conditions for its consumption and domestic use. According to a study [1], 28\% of the rural population in Colombia lacks an aqueduct, indicating that there is direct consumption of water sources without adequate chemical and microbiological treatment. In addition, according to the National Institute of Health in 2015 [2], only $15.1 \%$ of Colombians use water in optimal conditions for human consumption, showing a public health problem that includes various diseases caused by microbiological contamination. The use of contaminated water with the presence of pathogenic microorganisms causes gastrointestinal diseases, parasitic diseases, and diarrhea; among others, it negatively impacts the quality of life of the population.
In Quindio Region in Colombia, a study reported positive samples for protozoan parasites such as Toxoplasma gondii (58.6\%), Cryptosporidium parvum (21\%), and Giardia $(18.3 \%)$ in drinkable water [1]. Infections caused by parasites imply a relevant public health problem because the oocysts of these microorganisms are resistant to chlorines and chloramines, substances used in the chemical treatment of water [3].

Additionally, T. gondii is a parasitic protozoan that causes toxoplasmosis in humans. This disease can be asymptomatic in immunocompetent persons; however, it is a cause of cerebral toxoplasmosis in immunocompromised persons and in pregnant women, and it can cause alterations in the fetus, such as hydrocephalus, microcephaly, and chorioretinitis; among others, the more serious alteration depends on the type of organism [4]. This parasite is highly prevalent in Colombia (47.1\%) [5], especially given that it is 
spread in many different ways, for example, through the consumption of oocysts present in vegetables and drinkable water [6], as well as tissue cysts present in meat [1].

The oocystic state of $T$. gondii is very resistant to environmental conditions. It survives for approximately 1 year in soil [7] and 54 months in water [1], maintaining its processes of proliferation and infection. Dumètre in 2003 reported resistance of oocysts to different purification treatments of water with chlorinated products. The results showed the presence of the microorganism in the treated samples demonstrating its high capacity to stay in environmental conditions to develop its biological life cycle and become developmental stage more infectious at the epidemiological level [6].

T. gondii is characterized by its resistance to water purification processes, which evidences the search of other alternatives for treatment and disinfection of this resource. There are several methods that affect the $T$. gondii parasite, for example, the cooking of foods at temperatures above $71^{\circ} \mathrm{C}$ or the conservation of them at temperatures below $-20^{\circ} \mathrm{C}$ [8]; however, significant changes in the nutritional composition of some foods can occur in these temperature ranges [9]. Considering these limitations, other water purification alternatives have been proposed with the use of silver nanoparticles (AgNPs) due to their antimicrobial properties $[10,11]$. Currently, several studies describing procedures for the separation of T. gondii oocysts from water and soil samples are reported using immunomagnetic nanoparticles under laboratory conditions [12]. Another study reports preliminary results of the affectation of $T$. gondii oocysts through of UV radiation, showing morphological damage to the structure of the oocyst [13].

Another microorganism established as a water contaminant is Salmonella braenderup, a Gram-negative bacteria characterized by being found in contaminated water sources; a study conducted in Itagüí, Colombia, showed a high prevalence of S. braenderup in treated water [14] being one of the major causes of diseases such as gastroenteritis and typhoid fever in people. In addition, various species of Salmonella have developed resistance to antibiotics, making it an important public health problem [15].

Currently, the use of nanotechnology in water filtration systems for human consumption is increasing. The AgNPs have been projected as a potential material with a biocidal effect due to their antimicrobial properties. Some studies report new water filtration systems incorporating silver nanoparticles to polymer membranes, which demonstrated their effectiveness as biocides and potential inhibition to the formation of biofilms [16]. Other studies report important applications of the use of AgNPs to affect protozoan parasites pathogenic for human health and that present mechanisms of resistance to chlorine treatments. Cameron et al. demonstrated in their study breakdown of the cell structure of oocysts Cryptosporidium parvum when exposed to AgNPs, revealing a significant decrease in the viability of the oocysts of the parasite [10].

In this work, we evaluated effect of $10 \mathrm{~nm}$ silver nanoparticles (Sigma-Aldrich) as an alternative to affect $T$. gondii oocysts and Salmonella braenderup, developing a protocol- specific treatment in which conditions of agitation time and the proportion of the mixture between AgNPs and the biological material were varied.

\section{Materials and Method}

2.1. Materials. In this work, all safety precautions were used when working with biological material such as $T$. gondii oocysts [17]. In this study, silver nanoparticles (AgNPs) of $10 \mathrm{~nm}$ and concentration of $20 \mu \mathrm{g} / \mu \mathrm{l}$ (Sigma-Aldrich Co. LLC) were used. The MARTIN ultrafiltration membranes for vacuum filtration left immersed in distilled water for 48 hours (MARTIN membranes were obtained from the company MARTIN Membrane Systems, Germany). The oocysts from Toxoplasma gondii (were obtained from GEPAMOL group, Quindío University, Colombia) were stored in PBS $1 \mathrm{X}$ (phosphate-buffered saline) at $4^{\circ} \mathrm{C}$, and all the experiments were developed within 4 months of oocyst preparation. Gram-negative bacteria, Salmonella braenderup (it was obtained from LGMB group, Universidad El Bosque, Colombia), were cultured in a Luria-Bertani (LB) medium and incubated at $37^{\circ} \mathrm{C}$.

2.2. DNA Isolation. DNA of the samples analyzed during all the treatments was extracted from oocysts of $T$. gondii, and the positive control DNA was extracted from T. gondii tachyzoites (the BBMP Group, Universidad de los Andes, Colombia) to obtain high-quality T. gondii DNA for the nested-PCR. $300 \mu \mathrm{L}$ of T. gondii oocysts in PBS 1X was used to extract DNA using the Genomic DNA Isolation kit (Norgen Biotek Corp.) according to the manufacturer's instructions. The final concentration of DNA was measured with a NanoDrop 2000/c spectrophotometer (Thermo Scientific). The obtained genomic DNA was suspended in water molecular grade (Thermo Scientific) and stored at $-20^{\circ} \mathrm{C}$ for subsequent PCR processes.

2.3. Molecular Detection of T. gondii for PCR. Nested-PCR was performed to detect the $\mathrm{B} 1$ gen of $T$. gondii in initial samples used for the entire process with the AgNPs. Control and monitoring strategies were established to the specific norms to work with the PCR technique before the experimental work to avoid contamination. The cabinet was thoroughly cleaned and subsequently irradiated with UV for 30 minutes before use, and the materials used for the PCR, such as pipettes, PCR tubes, reaction vials, and pipet tips, are unique to this procedure and irradiated in the cabinet with UV for 20 minutes before being used. Also, sterile gloves and laboratory clothing were used exclusively for PCR work. In addition, filter tips were used for DNA extraction and PCR, and care was taken with pipetting to avoid contamination of the pipette cylinder. All the reagents used were molecular grade and were selected before use, and Taq polymerase was previously selected before use to guarantee a DNA-free process.

The two PCRs were worked under the same conditions, external primers $\mathrm{Pml} / \mathrm{S} 1$ and $\mathrm{Pml} / \mathrm{AS} 1$ and internal primers $\mathrm{Pml} / \mathrm{S} 2$ and Pml/AS2 [4]. One master mixture was prepared 
for PCR, were added $12.2 \mu \mathrm{l}$ of $\mathrm{H}_{2}$ (Odde), $2.5 \mu \mathrm{l}$ of dNTPs $(2 \mathrm{mM}), 1.5 \mu \mathrm{l}$ of forward primer, $1.5 \mu \mathrm{l}$ of reverse primer, $2.5 \mu \mathrm{l}$ of $\mathrm{MgCl}_{2}(17.5 \mathrm{mM}), 2.5 \mu \mathrm{l}$ of Dream Buffer, and $0.3 \mu \mathrm{l}$ of Taq polymerase. $23 \mu \mathrm{l}$ of the master mix and $2 \mu \mathrm{l}$ of DNA were added to each PCR tube. Thermal cycler conditions were as follows: 1 initial denaturation cycle at $95 \mathrm{C}$ for 3 minutes, 1 cycle of denaturation at $95 \mathrm{C}$ for 30 seconds, 39 cycles of alignment at $55.7^{\circ} \mathrm{C}$ for 30 seconds, 39 cycles of extension at $72^{\circ} \mathrm{C}$ per 1 minute, 1 cycle of final extension at $72^{\circ} \mathrm{C}$ for 15 minutes, and 1 final cycle at $12^{\circ} \mathrm{C}$. DNA fragments were separated through agarose gel electrophoresis in $1.0 \% \mathrm{w} / \mathrm{v}$ for $75 \mathrm{~min}$ at $100 \mathrm{v}$. A $1 \mathrm{kbp}$ DNA ladder (Thermo Scientific) was used.

2.4. Antimicrobial Performance of AgNPs with T. gondii Oocysts and Salmonella braenderup. T. gondii oocysts incubated without nanoparticles were used as control. The treatments with AgNPs were prepared in a ratio of $1: 4$ of oocysts (PBS 1X) and AgNPs $(20 \mu \mathrm{g} / \mu \mathrm{L})$ and then incubated at room temperature with constant agitation at $500 \mathrm{rpm}$ for 30, 45, and 60 minutes. For the evaluation of $S$. braenderup, samples of these bacteria incubated without nanoparticles were used as control. All samples were incubated at room temperature, constant agitation at $180 \mathrm{rpm}$, and different incubation times. Treatments were as follows: first treatment in a ratio of $1: 1$ of suspension $S$. braenderup and AgNPs, shaking for 1 hour and 2 hours; second treatment in a ratio of $1: 2$ of suspension $S$. braenderup and AgNPs, shaking for 1 hour and 2 hours; third treatment in a ratio of $1: 3$ of suspension $S$. braenderup and AgNPs, shaking for 2 hours; and fourth treatment in a ratio of $1: 4$ of suspension S. braenderup and AgNPs, shaking for $2 \mathrm{hrs}$.

The previous samples were separated in two aliquots to demonstrate morphological changes in the cell structure of the microorganisms, and some samples were analyzed by fluorescence microscopy and others by scanning electron microscopy (SEM).

2.5. Fluorescence Microscopy. Fluorescence microscopy was used to evaluate the morphological changes of Toxoplasma gondii oocysts and Salmonella braenderup treated with AgNPs and without treatment. Toxoplasma gondii oocysts and Salmonella braenderup not exposed to AgNPs were used as control. $50 \mu \mathrm{L}$ of each of the treatments and the control samples (no treatment) were mounted on slides previously washed with $40 \% \mathrm{HCl}$. Then, the organisms were rinsed by three times with phosphate-buffered saline (PBS) with added $\mathrm{CaCl}_{2}$ and $\mathrm{MgCl}_{2}$. After, the organisms were incubated in 4',6-diamidino-2-phenylindole (DAPI) labeling solution at $2 \mu \mathrm{g} / \mathrm{mL}$ for $15 \mathrm{~min}$ at room temperature. The labeling solution was aspirated. Then, the organisms were rinsed once more in PBS with added $\mathrm{CaCl}_{2}$ and $\mathrm{MgCl}_{2}$. Then, the coverslip was placed on each of the samples and these were sealed. Images were acquired in a Zeiss Axio imager A2 microscope with an excitation UV filter of $330-380 \mathrm{~nm}$ and emission $461 \mathrm{~nm}$.
2.6. Scanning Electron Microscopy (SEM). The size and morphology of Toxoplasma gondii oocysts and Salmonella braenderup exposed to treatment with AgNPs and without treatment were also examined using SEM microscopy. Toxoplasma gondii oocysts and Salmonella braenderup not exposed to AgNPs were used as control. The samples of $T$. gondii were centrifuged at $700 \times \mathrm{g}$ for 5 minutes and $S$. braenderup were centrifuged at $500 \times \mathrm{g}$ for 10 minutes. Then, each of the samples was vacuum filtered in MARTIN membranes, which were previously washed for 48 hours with distilled water. The membranes with $T$. gondii oocysts and $S$. braenderup were submitted to SEM, doing a previous preparation, initial washing with buffer PBS $1 \mathrm{X}$ for 10 minutes, then washing with $2.5 \%$ glutaraldehyde for 6 hours, $50 \%$ ethanol for 5 minutes, $70 \%$ ethanol for 10 minutes, $95 \%$ ethanol for 10 minutes, and $100 \%$ ethanol for 20 minutes. Finally, the samples were sent to INTEK GROUP laboratories for to perform SEM. The supernatant of one of the samples was placed on carbon tape and were also sent for to perform SEM to evaluate the reuse of the AgNPs.

\section{Results and Discussion}

3.1. Molecular Detection of T. gondii. The molecular detection of T. gondii was performed by PCR, identifying the B1 gen in the parasite samples. As seen in Figure 1, a $530 \mathrm{bp}$ amplicon corresponding to a fragment of the B1 gen of $T$. gondii was obtained, confirming that the samples used for the evaluation with silver nanoparticles contain oocysts of the parasite.

3.2. Morphology Alteration of T. gondii Oocyst and S. braenderup by Fluorescence Microscopy. The results generated in fluorescence microscopy demonstrate structural morphological changes in T. gondii oocysts and S. braenderup bacteria exposed to AgNPs compared to control (no treatment). Figure 2(a) shows sporulated oocysts of the control sample, which was not treated with AgNPs. The nonaltered sporulated oocysts of $T$. gondii have a subspherical to ellipsoidal morphology similar to which presented in a study by Ramakrishnan et al. in [18] with sizes between 11 and $13 \mu \mathrm{m}$ in diameter; also, its sporulated oocysts have thin a wall (arrow) and contain two ellipsoidal sporocysts (arrowhead) with sizes between 6 and $8 \mu \mathrm{m}$ as shown in Figure 2(a). Similar results are obtained by other studies, Dubey showed autofluorescence results of sporulated T. gondii oocysts under normal conditions [19], and each sporulated oocyst contains two ellipsoidal morphology sporocysts, with sizes from 6 to 8 um and a thin wall with complete integrity. Normal sporulated oocysts show subspherical to ellipsoidal morphology and are approximately $11 \times 13 \mu \mathrm{m}$ in diameter. Another study showed normal sporulated $T$. gondii oocysts like those shown in our results. The autofluorescence of these oocysts maintains a specific pattern of T. gondii, and the wall of the two sporocysts within the oocyst is clearly visible, showing its integrity, which allows easy detection of this protozoa parasite [20]. Ramakrishnan et al. [18] showed representative oocysts 


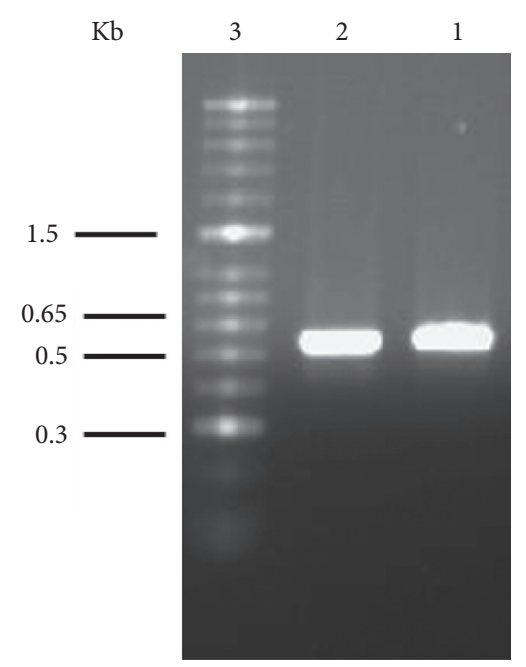

Figure 1: Nested-PCR of T. gondii B1 gen. (1) Control + T. gondii tachyzoites, RH strain. (2) T. gondii oocysts. (3) the DNA marker used. The primers amplified the expected $0.530 \mathrm{~kb}$ product in samples of T. gondii oocysts used for the evaluation of AgNPs with $1.0 \%$ agarose gel.

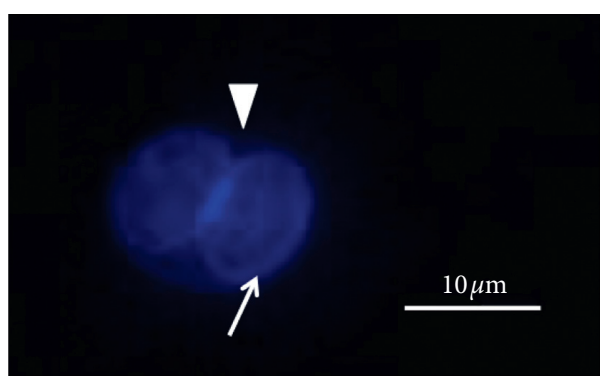

(a)

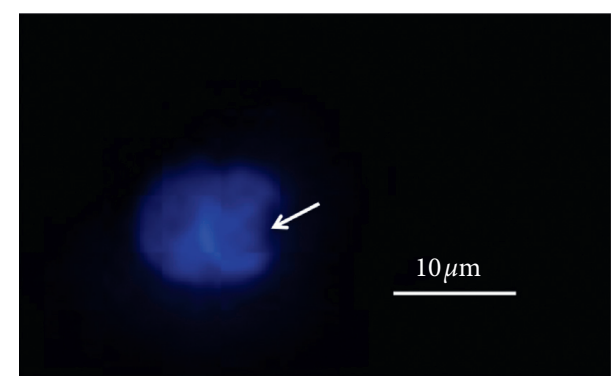

(b)

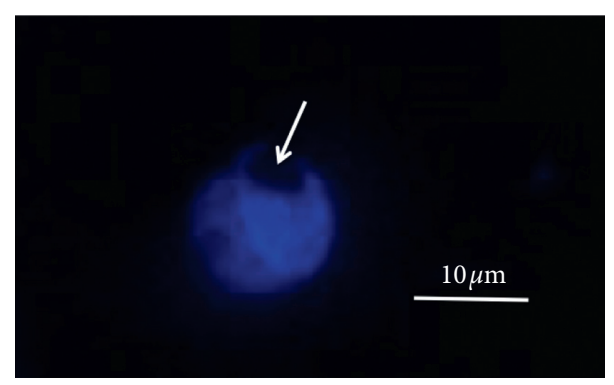

(c)

FIGURE 2: Effect of AgNPs in Toxoplasma gondii morphology as assessed by fluorescence microscopy. In the control sample, intact oocyst (a), in the presence AgNPs at $45 \mathrm{~min}$ (b) and $60 \mathrm{~min}$ (c).

exploiting the autofluorescence of the oocyst/sporocyst wall under the DAPI channel and using differential interference contrast settings, demonstrating that oocysts possess normal morphology and preserve their autofluorescence.

The treatments were realized varying the exposure times of the oocysts to the AgNPs, at $45 \mathrm{~min}$ with an agitation of $500 \mathrm{rpm}$, whose results are shown in Figure 2(b), an important morphological change is evidenced, and the oocyst which lost its ellipsoid shape and differences in the sizes of the sporocysts are observed as well as a break in the wall of one of the sporocysts (arrow) indicating alteration of the cell structure. Figure 2(c) shows samples at $60 \mathrm{~min}$ in agitation, the sporocysts are not clearly visible within the internal structure of the oocyst, this suggests alterations in the morphology of the oocyst, similar results are shown by Ramakrishnan et al. [18] where parasites deficient in HAP2 (HAP2 KO), an important protein in the regulation of the development of $T$. gondii within the intestine of the cat, presented oocysts that exhibit aberrant morphology and irregular and highly altered forms compared to normal oocysts. Also, in Figure 2(c), the rupture of the membrane that encloses one of the sporocysts is observed; however, in Figure 2(c), this alteration is pronounced more strongly than Figure 2(b) (arrow). 
Although studies on the effect of AgNPs on T. gondii oocysts are not reported in the literature, similar results are shown in Cryptosporidium parvum, a human pathogen of the phylum Apicomplexa such as T. gondii, and Pamela Cameron et al. evaluated the viability of oocysts exposed to AgNPs and silver ions [10], showed that both AgNPs and silver ions strongly decrease the viability of oocysts; however, the sporozoite/shell ratio was more affected with treatment with AgNPs possibly because sporozoites are destroyed by interaction with AgNPs.

Besides, fluorescence microscopy was performed to evaluate affectation of $S$. braenderup bacteria in the presence AgNPs. The results showed intensity decrease of fluorescence with DAPI staining and decrease in the number of bacterial population respect to control (Figures 3-5), and these changes are possibly due to the alteration of DNA integrity. Some studies report that AgNPs can anchor to the bacterial cell wall and interact with the cell membrane generating physical changes that allow AgNPs to penetrate into the intracellular environment. Furthermore, it has been shown that the antibacterial effect of AgNPs on Gramnegative bacteria such as Salmonella was stronger because the cell wall thickness of these bacteria is approximately 3-4 nm. AgNPs that achieve to enter the cell cytoplasm can release $\mathrm{Ag}+$ that can interact with various types of biomolecules such as proteins and DNA, leading to functional irregularity of the bacteria and subsequent cell death [21]. Furthermore, the antibacterial activity of AgNPs is related to the high production of reactive oxygen species (ROS) that can lead to DNA damage [22].

The histograms of the different treatments show a significant fluorescence decrease in relation to the increase in AgNP concentration and exposure time. This is clearly evidenced by the histogram in Figure 5(b) compared to control (Figure 6), where there is an absence of pixels with high intensities indicating a greater number of bacteria with low fluorescence.

In the control sample without AgNPs, high-intensity fluorescence was observed as presented in the histogram (Figure 6), while in the images obtained with the presence of AgNPs with different concentrations of AgNPs and exposure time, the bacteria reduced fluorescence intensity of DAPI (Figures 3(a) and 5(b)), which suggests changes in the integrity of the bacterial genome. Studies have found that $\mathrm{Ag}+\mathrm{can}$ be intercalated between the nitrogenous bases of nucleic acids generating the alteration of the $\mathrm{H}$-bonds and the formation of DNA strands, leading to the inhibition of cell division and the reproduction process [23].

The results determined by a concentration ratio of $1: 1, S$. braenderup and AgNPs, are shown in Figure 3(a). A less intense fluorescence was observed with respect to the control after 1 hour of exposure. The intensity was reduced in greater proportion at 2 hours of exposure (Figure 3(b)). By varying the concentration to $1: 2$, S. braenderup and AgNPs, it was observed that the intensity of the fluorescence was considerably reduced, indicating that the DNA was more affected in a greater number of cells at 1 hour and 2 hours of exposure (Figures 4(a) and 4(b)). This effect was more pronounced when the concentration was increased to $1: 3, S$. braenderup and AgNPs, with an exposure time of 2 hours (Figure 5(a)), where a faint fluorescence is observed in most cells. In the sample with a concentration ratio of $1: 4, S$. braenderup and AgNPs, at 2 hours of exposure (Figure 5(b)), a greater involvement of the nanoparticles was observed with a decrease in the intensity of the fluorescence of the bacteria, and this may suggest a possible bactericidal action of the AgNPs.

When comparing the action of the AgNPs to the same ratio of concentration and different exposure times, similar results are obtained, and Figures 3(a) and 3(b) show slight changes in fluorescence intensity at $1: 1$, the same ratio of AgNPs; however, it can be deduced that the variation in the exposure time of the bacteria to the AgNPs was not so significant, which may indicate that the AgNPs show significant results after one hour of application and this time could be enough to get bacteria affected at a ratio of $1: 1$. Similar results are shown in Figures 4(a) and 4(b), in a ratio of $1: 2$, which show slight changes in fluorescence intensity, and it can be deduced form these results that possibly one hour of exposure to AgNPs is enough time to obtain affectation of the bacteria at a ratio of $1: 2$. Figures $5(\mathrm{a})$ and 5(b) show significant changes in the fluorescence intensity of DAPI compared to the control. These results indicate that the increase in the concentration of AgNPs in the treatment for $S$. braenderup increases the antibacterial efficiency of AgNPs. The results show that the highest antibacterial activity was obtained in a concentration ratio of $1: 4$ at 2 hours of exposure. Some studies indicate that the efficiency in the antibacterial activity of AgNPs depends on the concentration used in the treatment [24]. Qing et al. reported that AgNPs show antibiofilm activity in treatments at concentrations up to $10 \mu \mathrm{g} / \mathrm{mL}$ [21]. Results of third and fourth treatments with AgNPs and Salmonella braenderup during $1 \mathrm{~h}$ are not shown because no significant changes were observed with respect to the control.

In relation to the bacterial density, we determined the number of bacteria per area from a preprocessing of the captured images, which consisted of the application of a Gaussian filter and then a threshold using Otsu's method, a well-known technique in image processing (Figure 7). This popular thresholding procedure has been widely implemented in the segmentation of fluorescent microscopy images [25]. Otsu thresholding technique searches for maximize variance between two classes (bacteria and background) by looking for the best value for the threshold in the global image, or in other word, it minimizes the overlap between the two components in the histogram [26]. As a result of applying this method, we obtained pixels separated into two classes, pixels with low or null fluorescence and pixels with significant fluorescence, and these correspond, respectively, to PBS (phosphate-buffered saline) and bacteria. This allowed determining the pixels corresponding to the bacteria and then generating a ratio between the cells and the area examined, and these results are shown in Figure 8. By comparing each treatment, it is shown that by increasing the concentration of AgNP and the time of exposure of the cells to the nanoparticles, the bacterial density decrease. The 1:4 treatment with 2 hours of exposure 


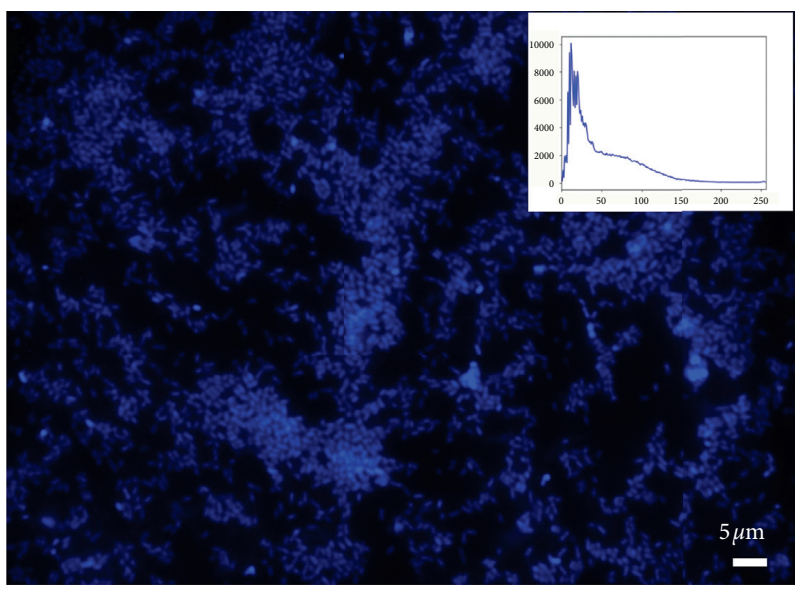

(a)

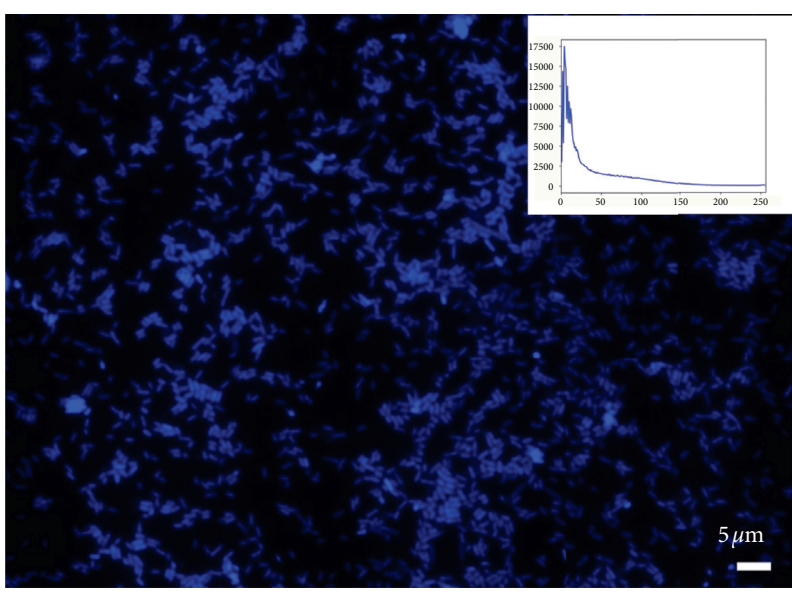

(b)

Figure 3: Effect of AgNPs in S. braenderup DNA integrity assessed by fluorescence microscopy with DAPI staining. In the presence of AgNPs, a concentration ratio of $1: 1$ for $1 \mathrm{hr}$ (a) and concentration ratio of $1: 1$ for $2 \mathrm{hrs}(\mathrm{b})$.

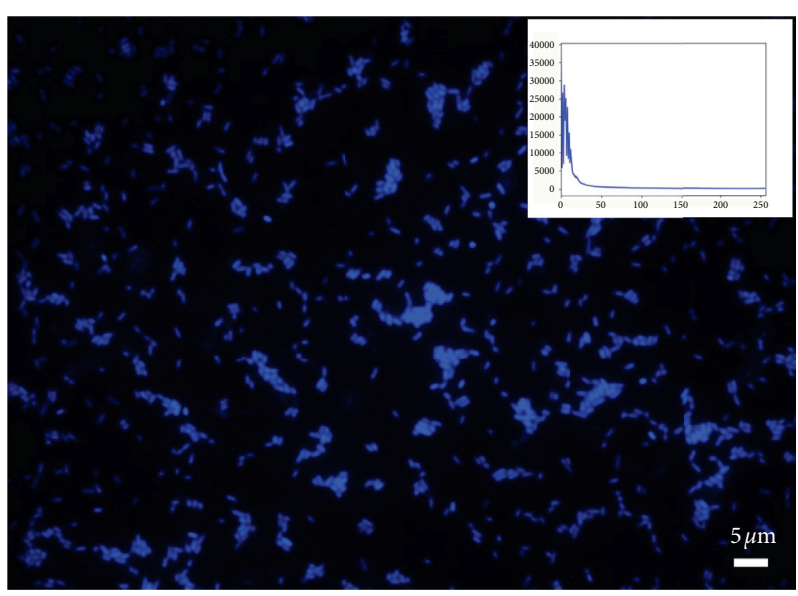

(a)

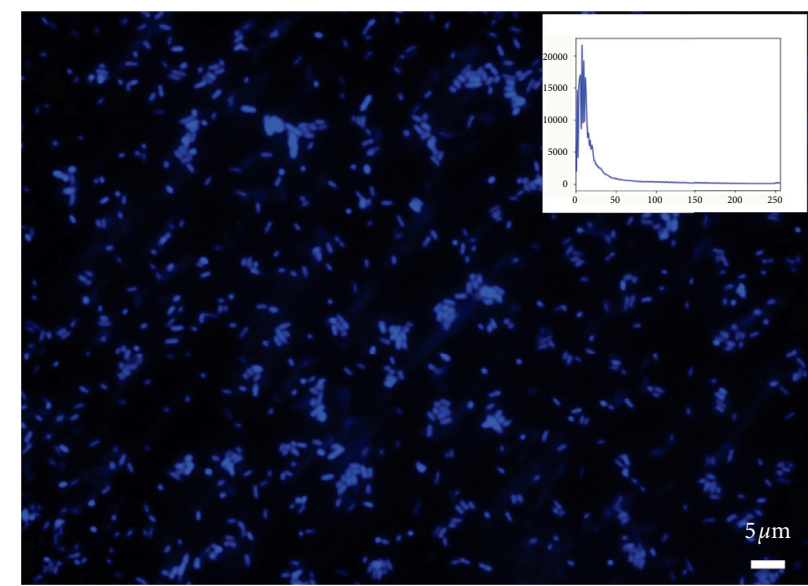

(b)

FIGURE 4: Effect of AgNPs in S. braenderup DNA integrity assessed by fluorescence microscopy with DAPI staining. In the presence of AgNPs, a concentration ratio of $1: 2$ for $1 \mathrm{hr}$ (a) and $1: 2$ for $2 \mathrm{hrs}(\mathrm{b})$.

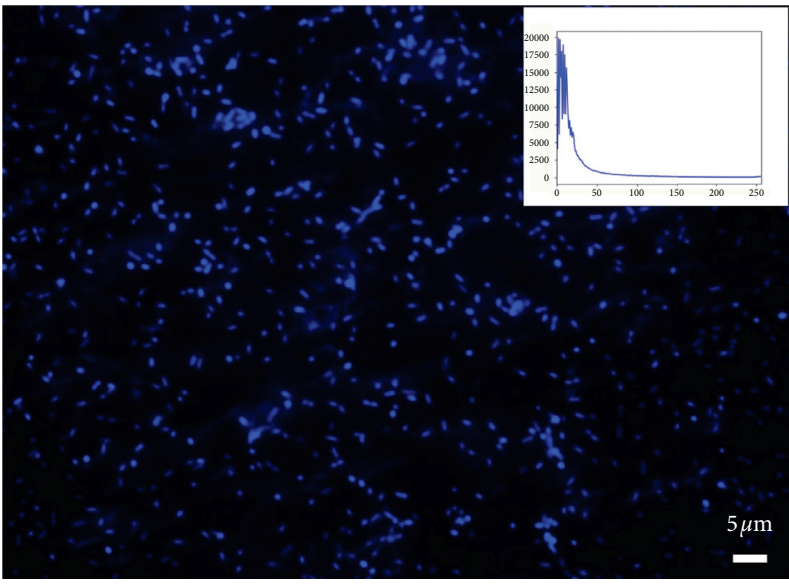

(a)

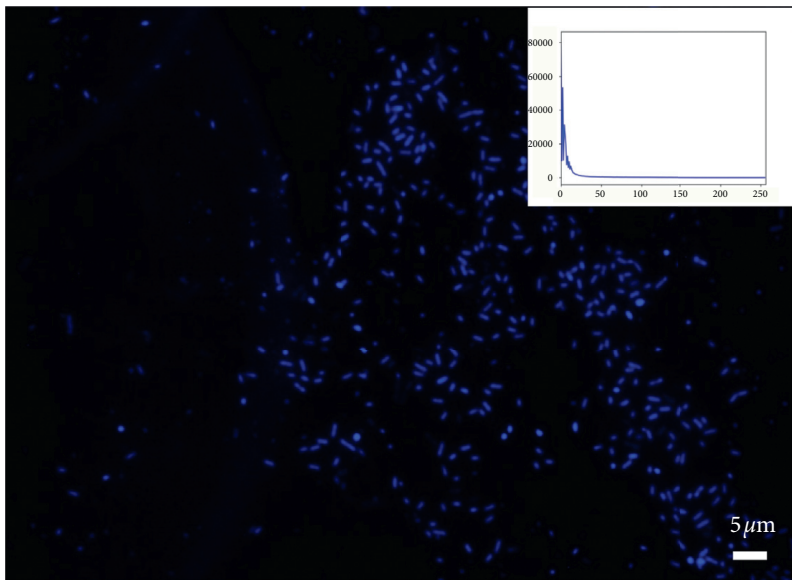

(b)

FIGURE 5: Effect of AgNPs in S. braenderup DNA integrity assessed by fluorescence microscopy with DAPI staining. In the presence of AgNPs, a concentration ratio of $1: 3$ for $2 \mathrm{hrs}(\mathrm{a})$ and $1: 4$ for $2 \mathrm{hrs}(\mathrm{b})$. 


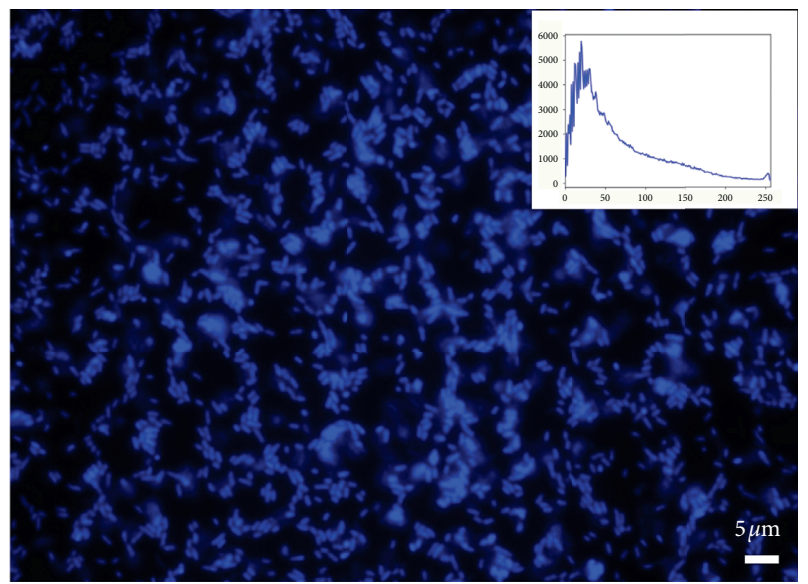

Figure 6: Cultures of $S$. Braenderup on the LB medium without AgNPs and stained with DAPI (control).

presented the lowest population density compared to the control group. This allows us to infer that the antimicrobial effectiveness of AgNPs can be achieved with this concentration and time of exposure.

Finally, these results suggest that the $1: 4$ ratio shows significant reduction of the bacterial population possibly due to DNA degradation, and these results show that the ratio 1 : 4 and 2 hours of exposure to AgNPs become the treatment with greater capacity to eliminate bacteria. For future studies, it is recommended to use specific dyes to evaluate cell viability.

\subsection{Scanning Electron Microscopy (SEM): Morphology Al-} teration of T. gondii Oocyst and S. braenderup. In the results obtained in SEM, a control sample of T. gondii oocysts was visualized without being exposed to the AgNPs and an oocyst was observed with its well-defined characteristic ellipsoid structure (Figure 9). Although many studies evaluating the oocyst morphology of $T$. gondii are not reported in the literature, one study showed morphological results similar to ours. They observed the ultrastructure of normal oocysts of $T$. gondii where they showed the ellipsoid shape and that the wall of the oocyst is a complex structure that has high resistance to physical and chemical changes which give it a solid appearance allowing the survival of the parasite [27]. When visualizing the oocysts by means of SEM after being exposed to the AgNPs, important structural changes were evidenced that indicate an impairment in their viability. Figure 10(a) shows oocysts of T. gondii with a time of exposure to AgNPs of $45 \mathrm{~min}$ in a concentration ratio of $1: 4$, demonstrating the destruction of the morphology of an oocyst with a size of $4.1 \mu \mathrm{m}$ in diameter, due to that lost its ovoid shape while breaking the wall of it. Not many studies are reported that evaluate the morphology of altered $T$. gondii oocysts; however, one study reported the results of ultrastructure of an oocyst showing the rupture of the wall with loss of the outer layer and part of the material of a disintegrated sporocyst [28]. Figure 10(b) shows oocysts with exposure time to AgNPs of $60 \mathrm{~min}$, showing significant

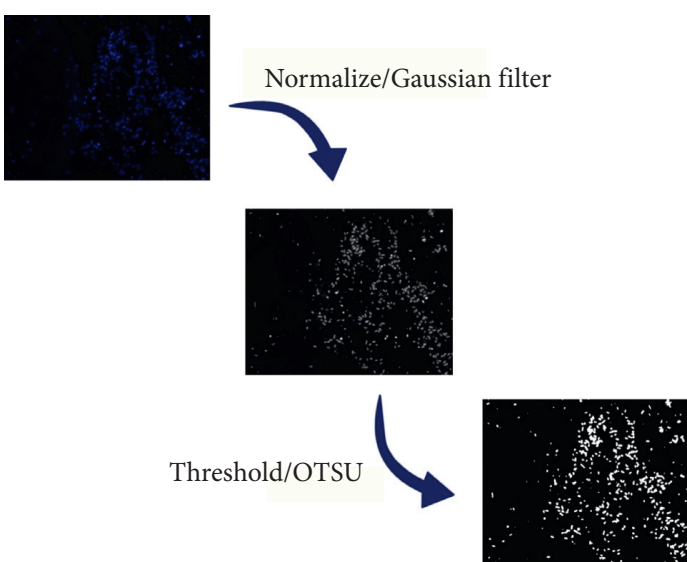

FIGURE 7: Image preprocessing to identify cell/area ratio.

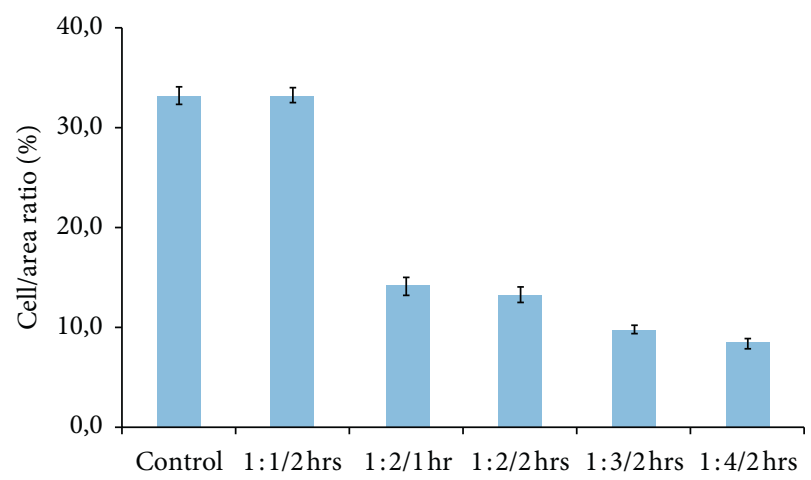

Figure 8: Percentage comparison of cell/area of the independent experiments (the data show results of independent replicates in each treatment).

destruction of the oocyst morphology, since its structure has more elongation and breaks in the wall.

Studies regarding the effect of AgNPs on Toxoplasma gondii oocysts are not reported in the literature. In fact, few studies are reported with morphological evaluation in developing stages of T. gondii treated with AgNPs. However, some studies show the anti-Toxoplasma activity of AgNPs in other stages of development of the parasite. A study by Shojaee et al. showed the effects of nanosilver colloid on tachyzoites and bradyzoites of T. gondii [29]. SEM results showed atypical and irregular morphology and presence of surface pores of tachyzoites of $T$. gondii after 2 hours of incubation with $20 \mathrm{ppm}$ of nanosilver colloid. Likewise, the nanosilver exposed cyst showed damage to the surface and bradyzoites with irregular and disorganized shapes were observed.

Other studies carried out with AgNPs in T. gondii show different alterations generated in the biological processes of the parasite. For example, the results of a study showed alteration in the conversion of T. gondii tachyzoite to bradyzoite, indicating that AgNPs reduce bradyzoites burden in vitro [30].

Alajmi et al. showed the anti-Toxoplasma gondii activity of silver nanoparticles by inhibiting inflammation processes 


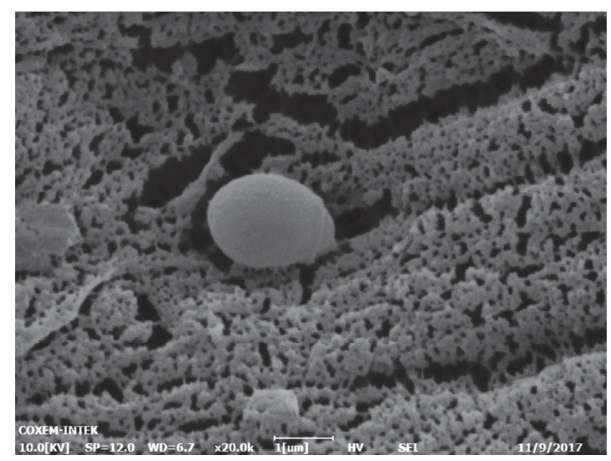

FIGURE 9: T. gondii oocyst morphology.

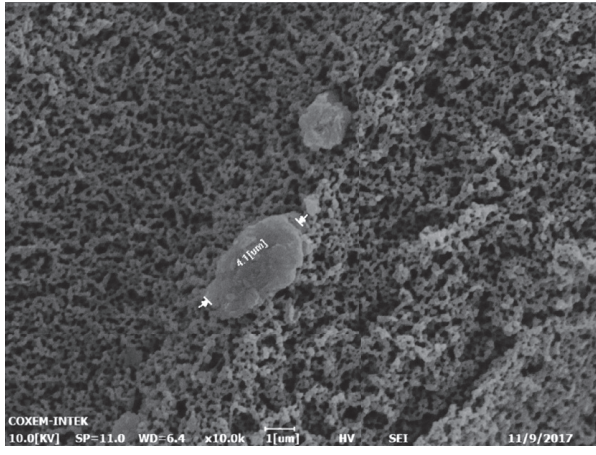

(a)

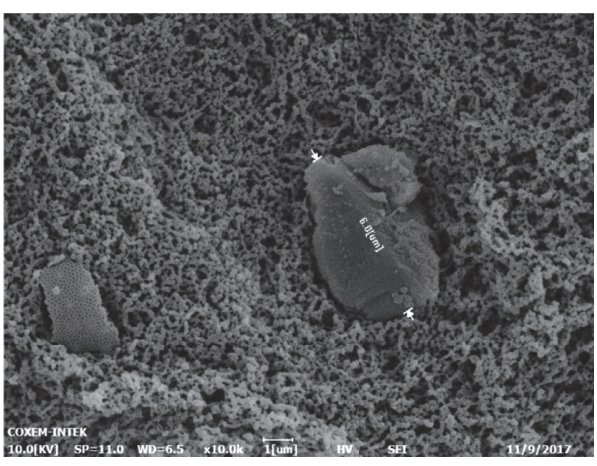

(b)

Figure 10: Effect of AgNPs in T. gondii oocyst morphology as assessed by SEM. In the control sample, intact oocyst (a), in the presence AgNPs for $45 \mathrm{~min}$ (b) and $60 \mathrm{~min}(\mathrm{c})$.

mediated by the regulation of cytokines [31]. Another study with inorganic nanoparticles including AgNPs showed antiparasitic activity for T. gondii, and the results showed that the potential of the parasite's mitochondrial membrane was affected, altering invasion, replication, and possible infectivity processes [32]. Although the mechanisms of action of AgNPs in the parasite Toxoplasma gondii are still unclear, studies reported in the literature indicate that AgNPs have a high potential for action in Toxoplasma gondii infection.

In the same way as the T. gondii oocysts, morphological changes were evidenced in S. braenderup assessed by SEM. A control sample of $S$. braenderup bacteria without exposure to AgNPs was visualized, where the characteristic structure of bacilli with sizes between $1.2 \mu \mathrm{m}$ and $1.3 \mu \mathrm{m}$ was shown (Figure 11). Similar results are obtained by SEM in which they showed images of Salmonella senftenberg under normal conditions. These bacteria belonging to the genus Salmonella have an elongated bacillus form. In these images, there is an appearance of clear texture on the surface of the bacterial cells and a smooth appearance on the cell walls. There is no alteration in the shape or size of the bacteria [33].

Figure 12(a) shows reduced sizes in the bacteria in the sample with a concentration ratio of $1: 2$ during $1 \mathrm{hr}$ of exposure; however, after 3 hours of exposure to the AgNPs,

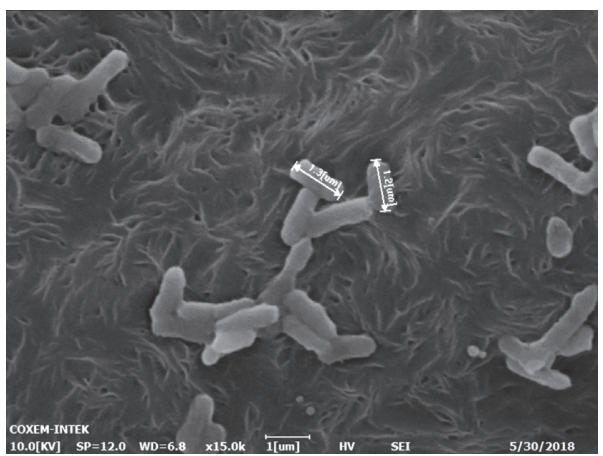

FIgURE 11: S. braenderup without presence of AgNPs (control). Morphology as assessed by SEM.

morphological alteration in the bacillus was observed, showing irregularities in the ends of the cell structure (Figure 12(a)). A study by AbuDalo suggests that although the antimicrobial mechanism of nanoparticles is still poorly understood, nanoparticles have the ability to interact with the cell wall of bacteria by altering lipopolysaccharides and forming pores that that alter the structure of the cell membrane [34].

In the samples with a concentration ratio of $1: 3, S$. braenderup and AgNPs, during 1 hour of exposure, changes in the size of the bacteria and in their morphology were evidenced (Figure 12(b)). Similar results were shown by 


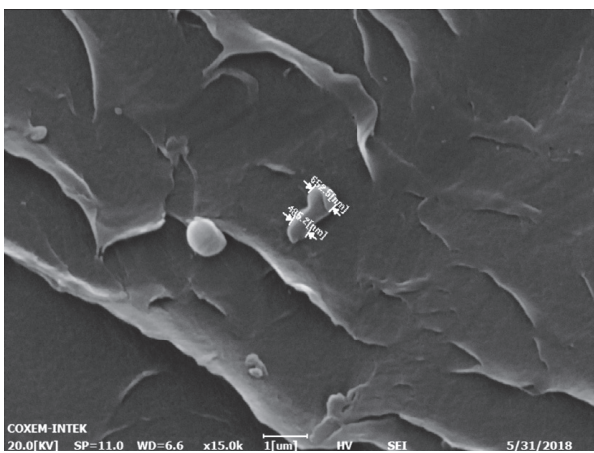

(a)

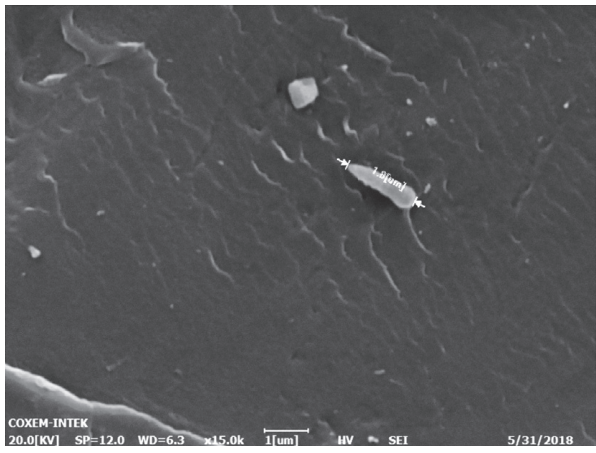

(c)

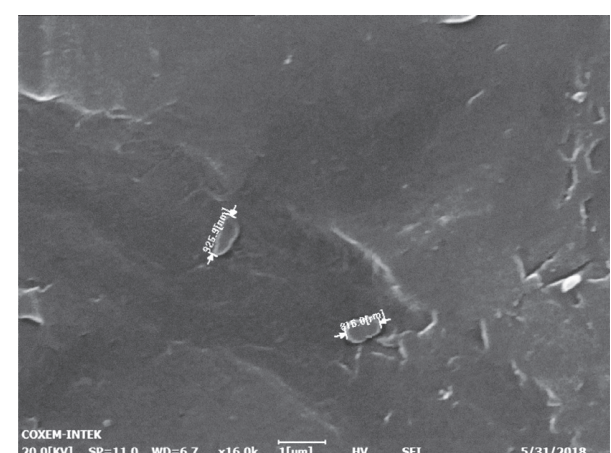

(b)

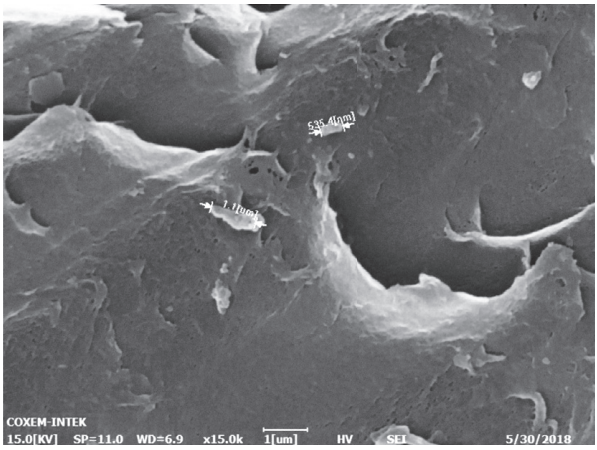

(d)

FigURE 12: Effect of AgNPs in S. braenderup morphology as assessed by SEM. In the presence of AgNPs, a concentration ratio of $1: 2$ for 3 hr (a), $1: 3$ for $1 \mathrm{hr}$ (b), $1: 3$ for $2 \mathrm{hrs}$ (c), and $1: 4$ for $2 \mathrm{hrs}$ (d).

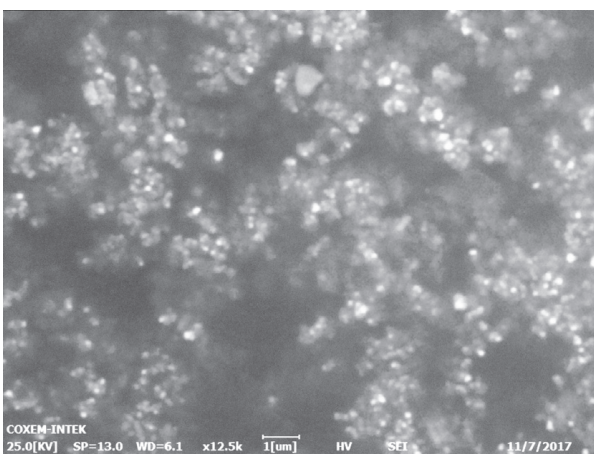

(a)

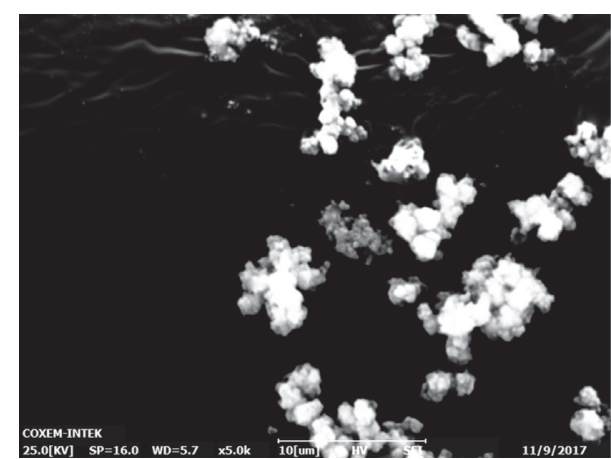

(b)

FIGURE 13: Image of AgNPs as assessed by SEM. AgNPs added to the oocyst and bacteria (a). AgNPs recuperated after of exposure to oocyst (b).

Neethu et al. on Salmonella typhimurium [35]. The SEM and TEM results showed the bactericidal activity of AgNPs using green synthesis with $P$. polonicum fungi. SEM visualization showed morphological damage, including deep craters and disintegrated cell membrane after 1 hour of treatment. The TEM results showed that after 30 minutes of exposure to AgNPs, the cells presented rupture in the cell wall and condensed cytoplasm and also complete bacterial lysis was observed at $24 \mathrm{hrs}$, suggesting that the cell wall of Salmonella typhimurium is permeable to AgNPs. At an exposure time of 2 hours, with the same 1:3 ratio, S. braenderup and AgNPs, more significant changes were observed than in Figure 12(b), since some of the bacteria have a more elongated structure
(Figure 12(c)). Some studies reported that the morphological changes presented in some bacteria exposed to AgNPs are due to Ag+ ions released from AgNPs that can bind to proteins that are present in the cell membrane, leading to protein inactivation and subsequent alteration of cell permeability [21]. Finally, in the samples with a ratio of $1: 4, S$. braenderup and AgNPs, during 2 hours of exposure, a significant decrease in the size of the bacteria was observed as well as morphological damages (Figure 12(d)). Although no studies evaluate the effect of AgNPs on Salmonella braenderup are reported in the literature, studies are found on other Salmonella species pathogenic for humans. A study reported by Vazquez-Muñoz [36] evaluated the antibacterial 
effect of AgNPs in Salmonella typhimurium. The results of cellular morphological visualization with TEM showed that AgNPs generate significant changes in the integrity of the bacterial cell membrane, suggesting a direct effect of silver ions on membrane stability.

Figure 13(a) shows AgNPs used in this work (Sigma) added to the oocyst and bacteria, where aggregates of nanoparticles are observed. Similar results are presented by AbuDalo, 2019 [34], in which images obtained by ESEM showed AgNPs with a spherical shape and average core sizes of 38 to $45 \mathrm{~nm}$. Furthermore, these AgNPs showed strong antibacterial activity against different species of bacteria. Figure 13(b) shows leftover AgNPs after exposure to $T$. gondii oocysts. AgNPs were examined using SEM sizes below $100 \mathrm{~nm}$ in diameter were obtained. This nanomaterial could be analyzed in other studies to confirm if they still maintain their antimicrobial properties.

\section{Conclusion}

The results obtained by the exposure of Salmonella braenderup and the Toxoplasma gondii oocysts to AgNPs showed pronounced morphological changes, which is an indicator of rupture in the bacillus membrane or in the wall of the oocyst, and this can be directly related to viability and the potential for infection of both microorganisms. Also other studies showed antibacterial activity against Gram-negative bacteria such as Salmonella and E. coli and Gram-positive bacteria such as Staphylococcus aureus and Bacillus subtilis, suggests that the AgNPs bind to the cell wall of the bacteria, causing membrane permeability and damaging vital structures of the bacteria, such as proteins, DNA, and enzymes [37].

Also Singh et al. reported that AgNPs showed antimicrobial activity against different species of bacteria, including Salmonella enterica [38], and AbuDalo also showed that AgNPs had an antibacterial effect against pathogens such as Salmonella enterica, Escherichia coli, and Staphylococcus aureus [34]. It is important to say that the variety in shapes and properties of various nanoparticles are characteristics responsible for the differences that occur regarding the antibacterial effect [21].

Additionally, it is possible to conclude that the results suggest a longer time of agitation of the mixture containing S. braenderup and T. gondii with AgNPs and significant morphological changes are evidenced, as it is observed in Figures 2(c), 3(b), 4(b), 5(a), 5(b), 10(b), 12(a), 12(c), and $12(d)$. Significant results are also shown in those treatments where there is a higher concentration of AgNPs, as shown in Figures 5(b) and 12(d). The results obtained allow deducing that there is a direct relationship between these two variables and the level of damage suffered by the microorganisms studied. This morphological destruction could be observed due to changes in morphology suffered by oocysts and bacteria, which also led to changes in size. Likewise, the results indicated that a certain amount of AgNPs was recoverable after a filtration process and can be potentially reusable after this process.

Finally, it is possible to conclude that the AgNPs have a high potential for reducing possibly the viability of oocysts of
T. gondii and S. braenderup bacteria. It is recommended in future to study the effect of different agitation speed and contact between AgNPs and microorganisms, which allows to detect changes at the level of the plasma membrane or the wall of the oocyst and bacteria, because certain levels of agitation allow a greater contact of the nanoparticles and, therefore, a more pronounced morphological change would be expected.

\section{Data Availability}

All data have been provided in the manuscript. For any further data that may be needed, contact the corresponding author.

\section{Conflicts of Interest}

The authors declare that they have no conflicts of interest.

\section{Acknowledgments}

The authors are grateful to the Research Vice-Rectory of Universidad El Bosque for their funding for the realization of this project (PCI-2016-9006). Acknowledgments to the Bioengineering Program of Universidad El Bosque and to the research groups LGMB (Universidad El Bosque), GEPAMOL (Quindío University), and BBMP (Andes University) for their valuable collaboration in the acquisition of biological material.

\section{References}

[1] J. Triviño-Valencia, J. E. Lora, J. D. Zuluaga, and J. E. GomezMarin, "Detection by pcr of pathogenic protozoa in raw and drinkable water samples in Colombia," Parasitology Research, vol. 115, no. 5, pp. 1789-1797, 2016.

[2] Ministerio de Salud y Protección Social, Informe nacional de calidad del agua para consumo humano INCA 2015, Ministerio de Salud y Protección Social, Bogota, Colombia, 2016.

[3] J. A. Castro-Hermida, M. González-Warleta, and M. Mezo, "Cryptosporidium spp. and giardia duodenalis as pathogenic contaminants of water in galicia, Spain: the need for safe drinking water," International Journal of Hygiene and Environmental Health, vol. 218, no. 1, pp. 132-138, 2015.

[4] D. K. Howe, S. Honoré, F. Derouin, and L. D. Sibley, "Determination of genotypes of Toxoplasma gondii strains isolated from patients with toxoplasmosis," Journal of Clinical Microbiology, vol. 35, no. 6, pp. 1411-1414, 1997.

[5] W. A. Cañón-Franco, N. López-Orozco, J. Gómez-Marìn, and J. P. Dubey, "An overview of seventy years of research (1944-2014) on toxoplasmosis in Colombia, South America," Parasites \&Vectors, vol. 7, no. 1, p. 427, 2014.

[6] A. Dumètre and M.-L. Dardé, "How to detect Toxoplasma gondii oocysts in environmental samples?" FEMS Microbiology Reviews, vol. 27, no. 5, pp. 651-661, 2003.

[7] M. Wang, P. Meng, Q. Ye et al., "Detection of Toxoplasma gondii oocysts in soils in northwestern China using a new semi-nested pcr assay," BMC Veterinary Research, vol. 10, p. 238, 2014.

[8] F. Lora, H. Aricapa, J. E. Pérez et al., "Detección de Toxoplasma gondii en carnes de consumo humano por la técnica de 
reacción en cadena de la polimerasa en tres ciudades del eje cafetero," Infectio, vol. 11, pp. 117-123, 2007.

[9] L. M. Mocanda and L. Gualdrón de Hernandez, "Retención de nutrientes en la cocción, freído y horneado de tres alimentos energéticos," Revista de Investigación, vol. 6, no. 2, pp. 179187, 2006.

[10] P. Cameron, B. K. Gaiser, B. Bhandari, P. M. Bartley, F. Katzer, and H. Bridle, "Silver nanoparticles decrease the viability of cryptosporidium parvum oocysts," Applied and Environmental Microbiology, vol. 82, no. 2, pp. 431-437, 2016.

[11] N. Durán, P. D. Marcato, R. D. Conti, O. L. Alves, F. T. M. Costa, and M. Brocchi, "Potential use of silver nanoparticles on pathogenic bacteria, their toxicity and possible mechanisms of action," Journal of the Brazilian Chemical Society, vol. 21, no. 6, pp. 949-959, 2010.

[12] J. L. Jones and J. P. Dubey, "Waterborne toxoplasmosis-recent developments," Experimental Parasitology, vol. 124, no. 1, pp. 10-25, 2010.

[13] A. Dumètre, C. Le Bras, M. Baffet et al., "Effects of ozone and ultraviolet radiation treatments on the infectivity of Toxoplasma gondii oocysts," Veterinary Parasitology, vol. 153, no. 3-4, pp. 209-213, 2008.

[14] K. Bedoya, C. Peláez, J. Acevedo, and S. Agudelo, "Caracterización de biosólidos generados en la planta de tratamiento de agua residual san fernando, itagüí (antioquia, Colombia)," Revista de Salud Pública, vol. 15, no. 5, pp. 778-790, 2013.

[15] K. O. Akinyemi, B. A. Iwalokun, F. Foli, K. Oshodi, and A. O. Coker, "Prevalence of multiple drug resistance and screening of enterotoxin (stn) gene in salmonella enterica serovars from water sources in lagos, Nigeria," Public Health, vol. 125, no. 2, pp. 65-71, 2011.

[16] S. Maharubin, Y. Zhou, and G. Z. Tan, "Integration of silver nanoparticles and microcurrent for water filtration," Separation and Purification Technology, vol. 212, pp. 57-64, 2019.

[17] S. E. Staggs, M. J. See, J. P. Dubey, and E. N. Villegas, "Obtaining highly purified Toxoplasma gondii oocysts by a discontinuous cesium chloride gradient," Journal of Visualized experiments: JoVE, vol. 33, p. 1420, 2009.

[18] C. Ramakrishnan, S. Maier, R. A. Walker et al., "An experimental genetically attenuated live vaccine to prevent transmission of Toxoplasma gondii by cats," Scientific Reports, vol. 9, p. 1474, 2019.

[19] J. P. Dubey, Toxoplasmosis of Animals and Humans, CRC Press, Boca Raton, FL, USA, 2nd edition, 2010.

[20] H. D. A. Lindquist, J. W. Bennett, J. D. Hester, M. W. Ware, J. P. Dubey, and W. V. Everson, "Autofluorescence of Toxoplasma gondii and related coccidian oocysts," Journal of Parasitology, vol. 89, no. 4, pp. 865-867, 2003.

[21] Y. A. Qing, L. Cheng, R. Li et al., "Potential antibacterial mechanism of silver nanoparticles and the optimization of orthopedic implants by advanced modification technologies," International Journal of Nanomedicine, vol. 13, pp. 3311-3327, 2018.

[22] R. Zhao, M. Lv, Y. Li et al., "Stable nanocomposite based on PEGylated and silver nanoparticles loaded graphene oxide for long-term antibacterial activity," ACS Applied Materials \& Interfaces, vol. 9, no. 18, pp. 15328-15341, 2017.

[23] D. W. Hatchett and H. S. White, "Electrochemistry of sulfur adlayers on the low-index faces of silver," The Journal of Physical Chemistry, vol. 100, no. 23, pp. 9854-9859, 1996.

[24] M. K. Rai, S. D. Deshmukh, A. P. Ingle, and A. K. Gade, "Silver nanoparticles: the powerful nanoweapon against multidrug- resistant bacteria," Journal of Applied Microbiology, vol. 112, no. 5, pp. 841-852, 2012.

[25] R. A. Russell, N. M. Adams, D. A. Stephens, E. Batty, K. Jensen, and P. S. Freemont, "Segmentation of fluorescence microscopy images for quantitative analysis of cell nuclear architecture," Biophysical Journal, vol. 96, no. 8, pp. 33793389, 2009.

[26] N. Otsu, "A threshold selection method from gray-level histograms," IEEE Transactions on Systems, Man, and Cybernetics, vol. 9, no. 1, pp. 62-66, 1979.

[27] D. J. P. Ferguson and J. F. Dubremetz, "The ultrastructure of Toxoplasma gondii," in Toxoplasma gondii-The Model Apicomplexan: Perspectives and Methods, L. M. Weiss and K. Kim, Eds., Elsevier, London, UK, 2007.

[28] A. Dumétre, J. P. Dubey, D. J. P. Ferguson, P. Bongrand, N. Azas, and P.-H. Puech, "Mechanics of the Toxoplasma gondii oocyst wall," Proceedings of the National Academy of Sciences, vol. 110, no. 28, pp. 11535-11540, 2013.

[29] S. Shojaee, N. Firouzeh, H. Keshavarz, S. Jafarpour Azami, M. Salimi, and M. Mohebali, "Nanosilver colloid inhibits Toxoplasma gondii tachyzoites and bradyzoites in vitro," Iranian Journal of Parasitology, vol. 14, no. 3, pp. 362-367, 2019.

[30] O. S. Adeyemi, Y. Murata, T. Sugi, Y. Han, and K. Kato, "Nanoparticles show potential to retard bradyzoites in vitro formation of Toxoplasma gondii," Folia Parasitologica, vol. 66, 2019.

[31] R. A. Alajmi, W. A. Al-Megrin, D. Metwally et al., "Antitoxoplasma activity of silver nanoparticles green synthesized with phoenix dactylifera and ziziphus spina-christi extracts which inhibits inflammation through liver regulation of cytokines in balb/c mice," Bioscience Reports, vol. 39, no. 5, 2019.

[32] O. S. Adeyemi, Y. Murata, T. Sugi, and K. Kato, "Inorganic nanoparticles kill Toxoplasma gondii via changes in redox status and mitochondrial membrane potential," International Journal of Nanomedicine, vol. 12, pp. 1647-1661, 2017.

[33] C. G. Golding, L. L. Lamboo, D. R. Beniac, and T. F. Booth, "The scanning electron microscope in microbiology and diagnosis of infectious disease," Scientific Reports, vol. 6, no. 1, Article ID 26516, 2016.

[34] M. A. AbuDalo, I. R. Al-Mheidat, A. W. Al-Shurafat, C. Grinham, and V. Oyanedel-Craver, "Synthesis of silver nanoparticles using a modified tollens' method in conjunction with phytochemicals and assessment of their antimicrobial activity," PeerJ, vol. 7, Article ID e6413, 2019.

[35] S. Neethu, S. J. Midhun, M. A. Sunil, S. Soumya, E. K. Radhakrishnan, and M. Jyothis, "Efficient visible light induced synthesis of silver nanoparticles by penicillium polonicum ara 10 isolated from chetomorpha antennina and its antibacterial efficacy against salmonella enterica serovar typhimurium," Journal of Photochemistry and Photobiology B: Biology, vol. 180, pp. 175-185, 2018.

[36] R. Vazquez-Muñoz, A. Meza-Villezcas, P. G. J. Fournier et al., "Enhancement of antibiotics antimicrobial activity due to the silver nanoparticles impact on the cell membrane," PLoS One, vol. 14, no. 11, Article ID e0224904, 2019.

[37] R. Li, Y. Pan, N. Li et al., "Antibacterial and cytotoxic activities of a green synthesized silver nanoparticles using corn silk aqueous extract," Colloids and Surfaces A: Physicochemical and Engineering Aspects, vol. 598, Article ID 124827, 2020.

[38] H. Singh, J. Du, P. Singh, and T. H. Yi, "Extracellular synthesis of silver nanoparticles by pseudomonas sp. thg-ls1.4 and their antimicrobial application," Journal of Pharmaceutical Analysis, vol. 8, no. 4, pp. 258-264, 2018. 